\title{
Seismic performance analysis of lifeline systems
}

\author{
M. Faraji \& J. Kiyono \\ Graduated School of Engineering, Kyoto University, Japan
}

\begin{abstract}
Seismic risk assessment of lifelines is considerably more complicated than that of a single structure on account of the geographical spread of lifelines. Lifeline risk assessment requires knowledge about ground-motion intensities at multiple sites. This study considers the seismic performance analysis of lifeline network systems. Seismic damage estimates of the potable water and electric power networks of the Bam city will be calculated for the expected earthquake scenario and the results will be utilized to assess the performances of the topological models of the networks. The study will first include collection of lifeline utility network data in GIS format. Seismic damage analysis will be made by defining fragilities of the network components and the ground motions generated by the scenario earthquake. The damage estimates will be utilized in the post-seismic state of the networks for disaster management countermeasures and activities.

Keywords: lifelines, seismic performance, risk assessment, fragility curve, probabilistic.
\end{abstract}

\section{Introduction}

Lifelines are the systems that relate to daily life needs, like water-supply, power, telecom, traffic, gas-supply, sewage and heat-supply, and so on. Strong dependence on lifeline system is one of the distinctive characteristics of modern urban area. The issue of vulnerability of critical infrastructures has recently attracted considerable attention from both the academic and policy-making spheres. A systematic method for addressing risk assessment and risk management is the, so-called, Probabilistic Risk Analysis (PRA), which concerns the performance of a complex system in order to understand likely outcomes and its areas of importance. PRA has historically been developed for situations in which measured data about the overall reliability of a system are 
limited and expert knowledge is the next best source of information available. It is valuable because it does not only quantify the probabilities of potential outcomes and losses, but it also delivers reproducible and objective results. There are many obstacles for the implementation PRA. One of the main reasons is lack of input data [8].

\section{Lifeline performance after 2003 Bam earthquake}

Bam is a city in south east of Iran. The 2003 Bam earthquake with magnitude $\mathrm{Mw}=6.5$ destroyed most of the city of Bam and nearby villages. The earthquake was by far the most devastating earthquake in the history of the region around Bam. The maximum uncorrected accelerations recorded at Bam station were $0.82 \mathrm{~g}, 1.01 \mathrm{~g}$ and $0.65 \mathrm{~g}$ in the longitudinal, vertical and transverse directions, respectively.

The earthquake mainly affected power, water, and communication networks in the epicentral regions, fig. 1. Damaged substation of the power transmission system and numerous electrical transmission concrete poles in the Bam electric distribution system, caused blackouts in city within hours following the earthquake and power had not been restored for several days. Water systems in Bam and Baravat experienced heavy damage and the water supply was cut off for a long duration due to extensive pipe breaks. Bam water system, on the other hand, experienced major damage mainly because of the old asbestos cement distribution lines. There were several breaks in the water distribution systems and minor damage to deep wells. The elevated water tank in the old section of the city was severely damaged. Although Bam have not had gas distribution network during earthquake [4].

The damage to roads, bridges, railway and airport was minor. Many streets and most of the alleys were blocked after the earthquake due to debris from the damaged buildings. The airport was out of operation for a few hours after the earthquake due to damage to the airport control tower but later played a major role in the rescue and relief operations [3].
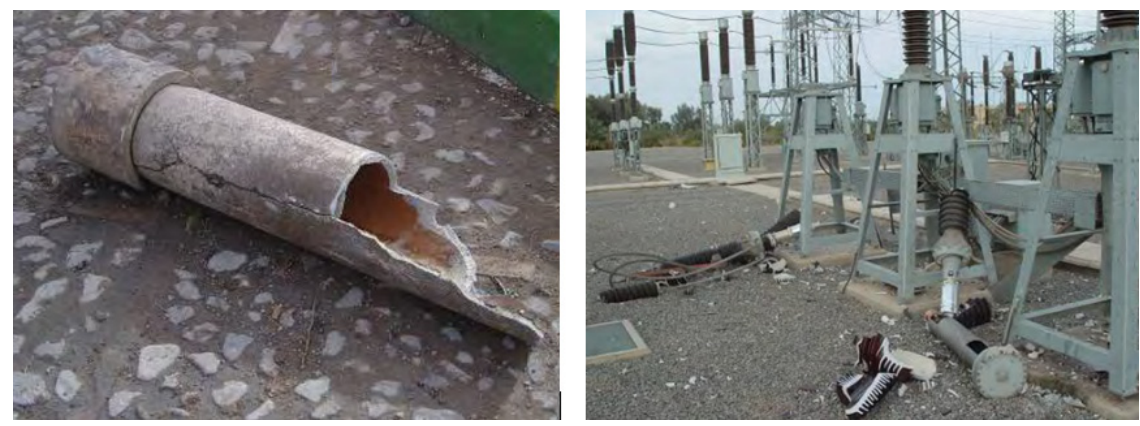

Figure 1: Damaged Bam asbestos cement water pipe and electric substation. 


\section{Seismic hazard analysis}

In order to assess risk to a structure from earthquake shaking, we must first determine the annual probability or rate of exceeding some level of earthquake ground shaking at a site, for a range of intensity levels [2]. The somewhat complicated probabilistic evaluation could be avoided if it was possible to identify a "worst-case" ground motion and evaluate the facility of interest under that ground motion. This line of thinking motivates an approach known as deterministic hazard analysis.

\subsection{Deterministic seismic hazard analysis}

The deterministic method is the standard approach in which effects from the largest earthquake expected (MCE) are the primary focus. Note that a scenario earthquake is suggested as the central concept for the 'deterministic' or 'maximum credible earthquake' in seismic hazard assessment [1].

The use of the MCE ensures that effects from all other magnitudes are explicitly considered. In other words, by virtue of designing a structure to withstand the MCE, it will automatically withstand all other (smaller) earthquakes. MCEs from all faults in the region are considered. Effects from all MCEs are compared, and the ones that would impact the most selected as the design earthquake. In the case of Bam city it is considered that Bam fault as a scenario earthquake, fig. 2. Bam fault caused the earthquake of 2003 is located $3 \mathrm{~km}$ far from city centre with magnitude 6.5 .

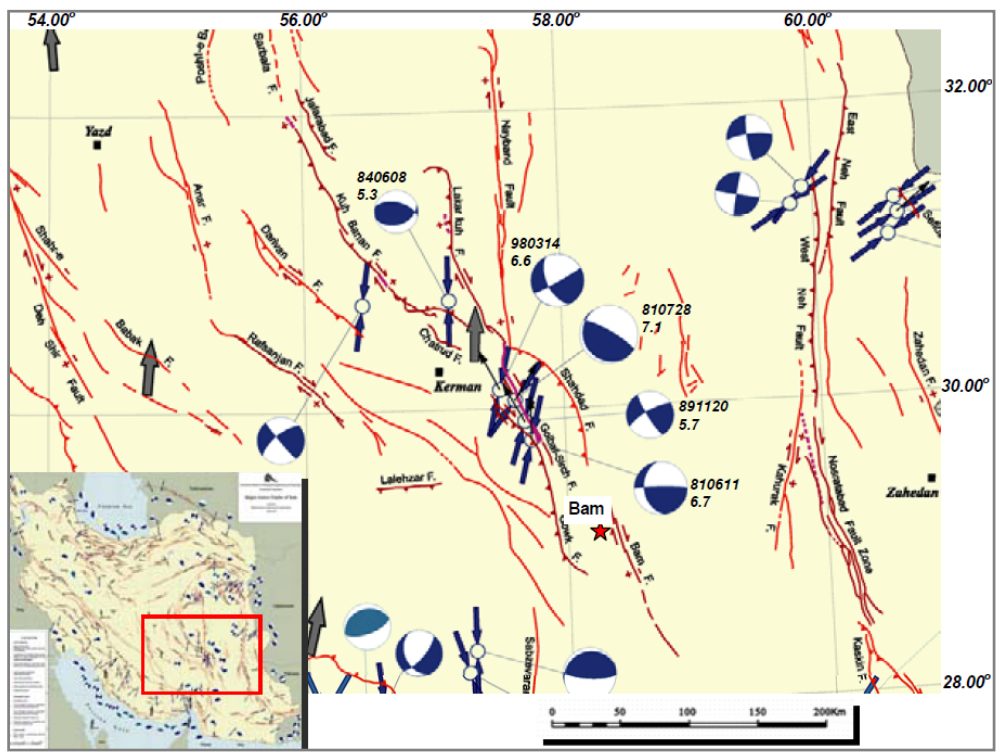

Figure 2: $\quad$ Regional seismology of Bam city (Courtesy of IIEES [7]). 


\subsection{Probabilistic seismic hazard analysis}

While the choice of a "worst-case" earthquake can be difficult and subjective, an even greater problem with deterministic hazard analysis is the choice of worstcase ground motion intensity associated with that earthquake. With probabilistic seismic hazard analysis (PSHA) it is no longer searching for elusive worst-case ground motion intensity. Rather, it will consider all possible earthquake events and resulting ground motions, along with their associated probabilities of occurrence, in order to find the level of ground motion intensity exceeded with some tolerably low rate [2]. At its most basic level, PSHA is composed of; Identify all earthquake sources capable of producing damaging ground motions, Characterize the distribution of earthquake magnitudes, Characterize the distribution of source-to-site distances associated with potential earthquakes, Predict the resulting distribution of ground motion intensity as a function of earthquake magnitude, distance, etc, and finally Combine uncertainties in earthquake size, location and ground motion intensity, using a calculation known as the total probability theorem.

\subsection{PSHA of Bam city}

It is interested in all earthquake sources capable of producing damaging ground motions at the site. These sources could be faults, which are typically planar surfaces identified through various means such as observations of past earthquake locations and geological evidence. If individual faults are not identifiable, then earthquake sources may be described by Areal Regions in which earthquakes may occur anywhere. Once all possible sources are identified, it can identify the distribution of magnitudes and source-to site distances associated with earthquakes from each source, fig. 3 .

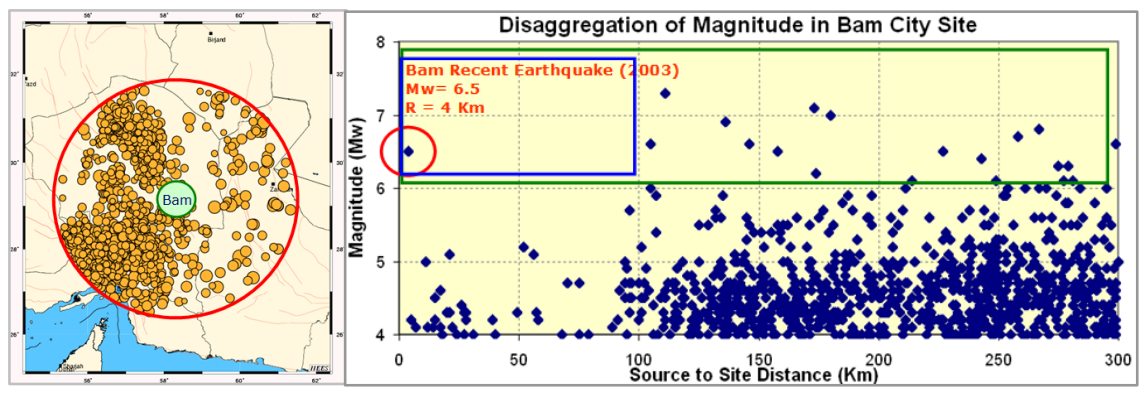

Figure 3: $\quad$ Earthquakes in southeast Iran (Epicentres from online IIEES).

In this study an areal source within a $300 \mathrm{~km}$ radius is considered that is capable of producing earthquakes with a variety of magnitudes. The area source produces earthquakes randomly and with equal likelihood anywhere within $300 \mathrm{~km}$ of the site. Areal sources are often used in practice to account for "background" seismicity, or for earthquakes that are not associated with any specific fault. The number of 1789 records was gathered. It is considered that the 
source produces events with $\mathrm{M} \geq 4$ ( 783 records) at a rate of 0.02 events per year. The distribution of those earthquakes follows the bounded GutenbergRichter model (eqn. (1)).

$$
\log \lambda_{m}=a-b m
$$

It is now quantified the distribution of potential earthquake magnitudes and locations, but we are interested in analyzing ground motions, not earthquakes.

Therefore it is considered a suitable ground motion prediction model depending on study area. Ghodrati et al. [6] proposed predictive model especially for Iran seismic zones, eqn. (2), for the mean of log peak ground acceleration (PGA) in units of gal and peak ground velocity (PGV) in units of kine [6].

$$
\ln y=C_{1}+C_{2} \cdot M_{s}+c_{3} \ln \left[R+C_{4} \exp \left(M_{s}\right)\right]+C_{5} R
$$

It is used total probability theorem, eqn. (3), to perform the PSHA calculation for PGA and PGV, using the Ghodrati et al. attenuation relation ground motion model. PGV and PGA hazard curve for Bam city is illustrated in fig. 3.

$$
\begin{gathered}
\lambda(I M>x)=\lambda\left(M>m_{\min }\right) \int_{m_{\min }}^{m_{\max }} \int_{0}^{r_{\max }} P(I M>x \mid m, r) \\
f_{M}(m) f_{R}(r) d r d m
\end{gathered}
$$

Where $\lambda(I M>x)$ is the rate of $I M>x, \lambda(M>m$ min) is the rate of occurrence of earthquakes greater than $m_{\text {min }}$ from the source and $f_{M}(m)$ and $f_{R}(r)$ are probability density functions for magnitude and distance, and it is integrated over all considered magnitudes and distances.

PSHA parameters, based upon topological properties taken from risk assessment, are computed and then visualised on a GIS. Lifeline systems are of large scale, complex and geographically distributed; use of GIS for the integration and manipulation of all available data has become more popular. Moreover, GIS plays a double role: in the first instance GIS software is a vital

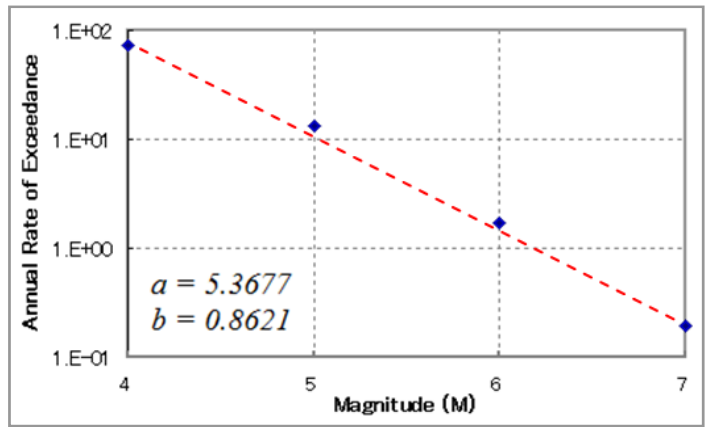

Figure 4: Distribution of observed earthquake magnitudes, along with eqn. (1). 


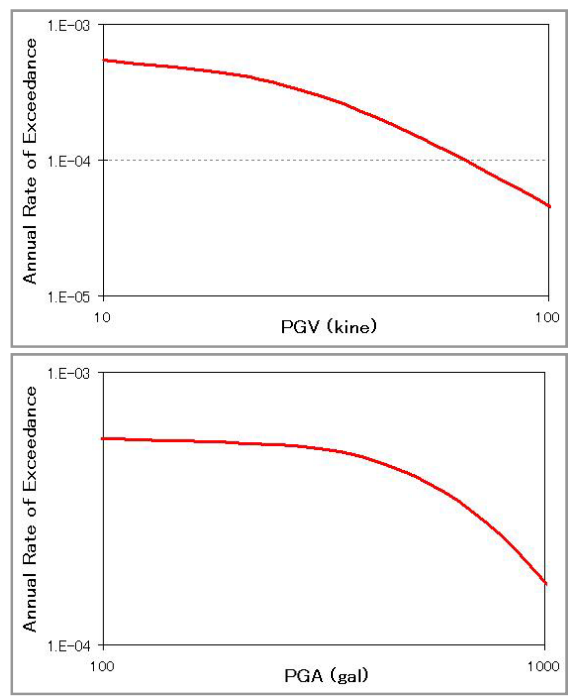

Figure 5: $\quad$ PGV and PGA hazard curve for Bam city, along with eqn. (3).

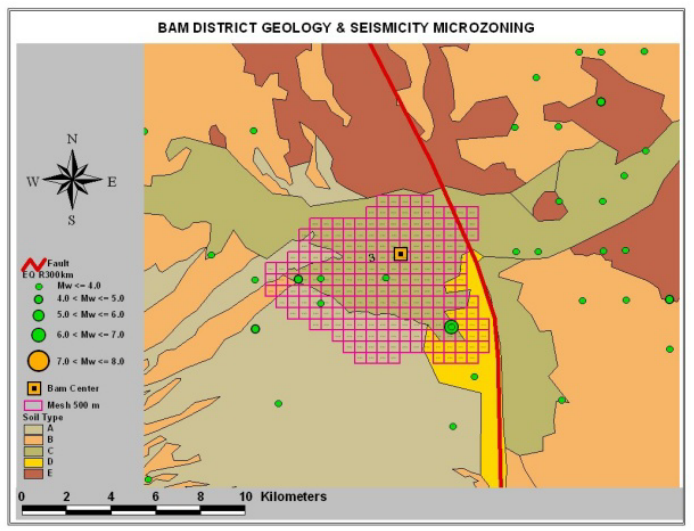

Figure 6: Bam district geology, seismicity and the city grid mesh in GIS.

tool for encompassing the spatial characteristics of infrastructure systems; and as such, it provides the topology of the network accompanied by additional information. Finally, having numerically processed, GIS can again be used for the effective visualization of results of the analysis in terms of various forms of mapping that allow users to examine spatial characteristics. Therefore, Bam district geology, seismicity and lifeline systems are modelled in GIS, fig. 6. Bam city is modelled by grid mesh $500 \mathrm{~m}$ dimension.

The description of the seismic hazard map is a monotonic function with the return period $T$ and the exposure time $n$. The return period (or recurrence interval) is the average time span between two events of a given magnitude at a particular site. The exposure time usually equals the expected life of the 
structure. In order to calculate the design life expectation of the structure, both these parameters (as well as the return period of the event) must be employed when calculating the risk of the structure with respect to a given event [5]. The risk assessment is thus the likelihood of at least one event that exceeds the design limits of the structure in its expected life. In this study, Bam seismic hazard maps calculated for 475 return period and 50 years of exposure time corresponds to $10 \%$ probability of exceedance, fig. 7 .

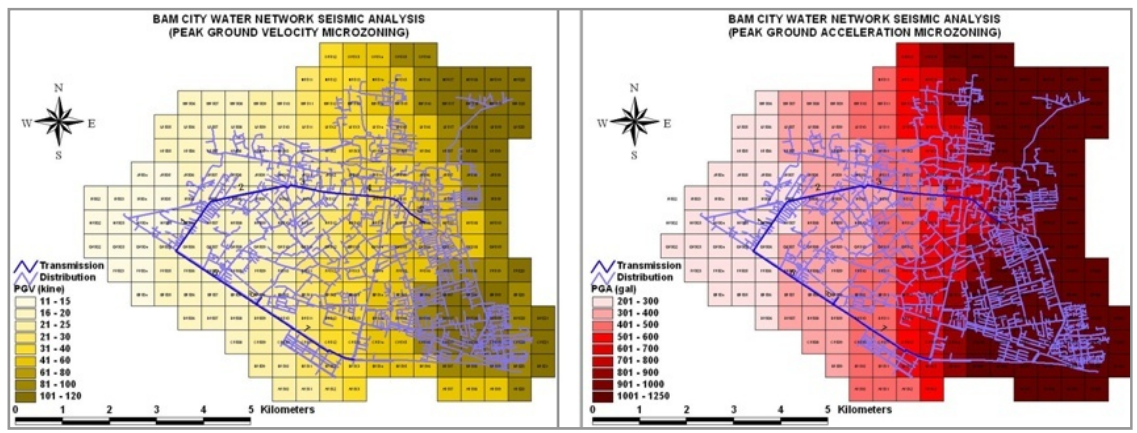

Figure 7: $\quad$ Bam PGV \& PGA hazard map calculated for 475 return periods.

In fact, there is $90 \%$ chance that these ground motions will not be exceeded. This level of ground shaking has been used for designing ordinary buildings in high seismic areas.

\section{Lifeline systems damage assessment}

The probability of physical damage to a facility is modeled by the seismic fragilities for various lifeline facilities. The damages of lifeline networks are usually showed by the correlation of damage ratio and earthquake parameter as fragility curves. Most researches of fragility curves are usually using $P G A$ or $P G V$ as earthquake parameter.

\subsection{Bam water network}

The Guidelines prepared by the American Lifeline Alliance suggests that damage to water pipe caused by strong ground motion can be expressed as a function of PGV, eqn. (4).

$$
R R=K(0.00187) P G V
$$

where RR is the repair ratio, which is the number of pipe breaks per 1000 feet (305 m) of pipe length, $\mathrm{K} 1$ is a coefficient determined by the pipe material, pipe joint type, pipe diameter and soil condition, and PGV has the units of in/sec. Generally water pipes installed in the Bam area were cement-asbestos and some of them were PVC and polyethylene. Only large-diameter water mains, with 
diameters from 10 inches to 25 inches $(250-600 \mathrm{~mm})$, were cast and ductile iron. Considering the typical water pipes, pipe material, pipe joint type, pipe diameter and soil conditions in Bam, it is assumed various $\mathrm{K} 1$ and calculated water network damage ratio in each $500 \mathrm{~m}$ grid mesh, fig. 8 .

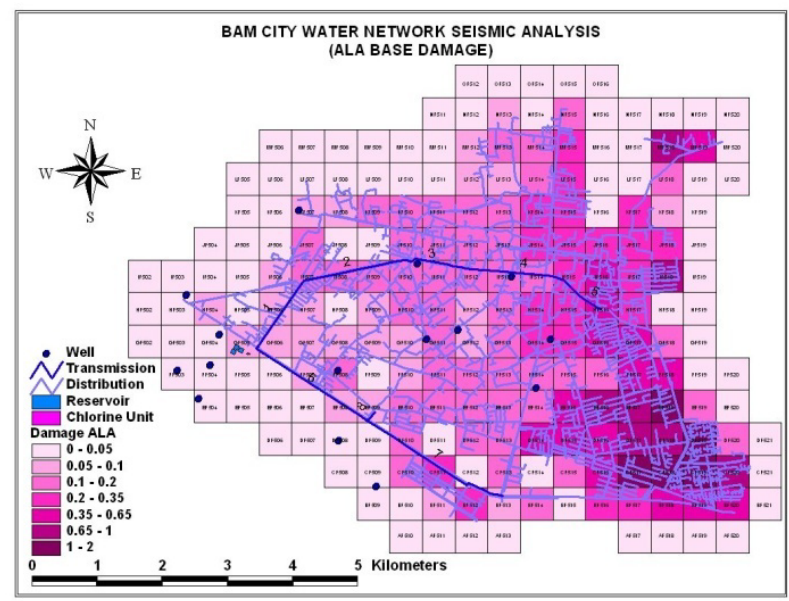

Figure 8: Bam water network damage ratio (based on ALA-2001).

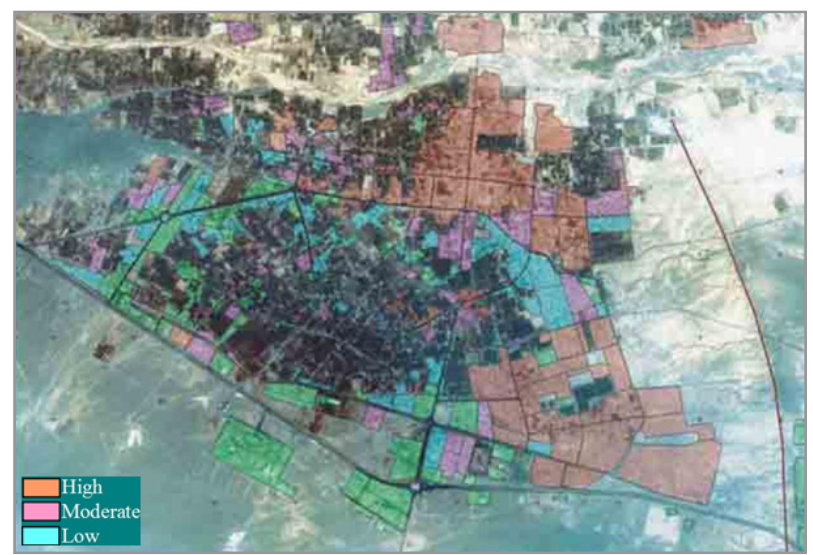

Figure 9: Bam buildings damage after earthquake (survey by JICA 2004).

Assuming that occurrence of one rupture impairs the pipeline functionality, fig. 1, and based on JICA study team report about building damage rate in Bam earthquake, as shown in fig. 9, the high damaged region in case of building is very similar to the region defined by high damage water pipelines, fig. 8. The fact that districts experiencing a high level of pipeline damage also experienced a high level of building damage implies that damage patterns were dominated by the influence of variations in strong motion from district to district. Therefore it 
seems that in case of network input data lack and without any lifeline damage assessment analysis, using the building damage database prepare suitable rough network damage estimation for disaster management and post earthquake activities such as emergency water supply.

\subsection{Bam electricity power network}

Electricity power network of Bam city is supplied by a $230 \mathrm{kv}$ transmission line. There is a 230/132 kv substation in the south part of the city, fig. 7. Bam power distribution network is about $600 \mathrm{~km}$ length and divided to two levels, medium $(20 \mathrm{kv})$ and low voltage $(220 \mathrm{v})$. Because of lack of data, in this study only the substation is considered to damage assessment.

In the case of electricity power system, it is used the fragility curves for the substations and power plants. The shape of the fragility curve for the given element is dependant on the damage state. More severe damage states correspond to the lower probability of exceedance at the same PGA. Damage states as defined in HAZUS are dependent on the type of element and the level of the damage of its subcomponents.

Fragility curves of the substations are classified according to the voltages assigned to the substation and according to whether all subcomponents of the substations are anchored or not. Substations are classified according to their voltage rating: from low voltage $(<150 \mathrm{kV})$, medium voltage $(150-350 \mathrm{kV})$ and high voltage $(>350 \mathrm{kV})$. Furthermore, we have to define the subcomponents of the substation. In case of Bam electricity power substation, it is considered medium voltage and unanchored. According to the location of the substation, affected PGA is about 574 gal. Thus by using medium voltage and unanchored fragility curve, fig. 10, the probability of physical damage is about 0.96 .

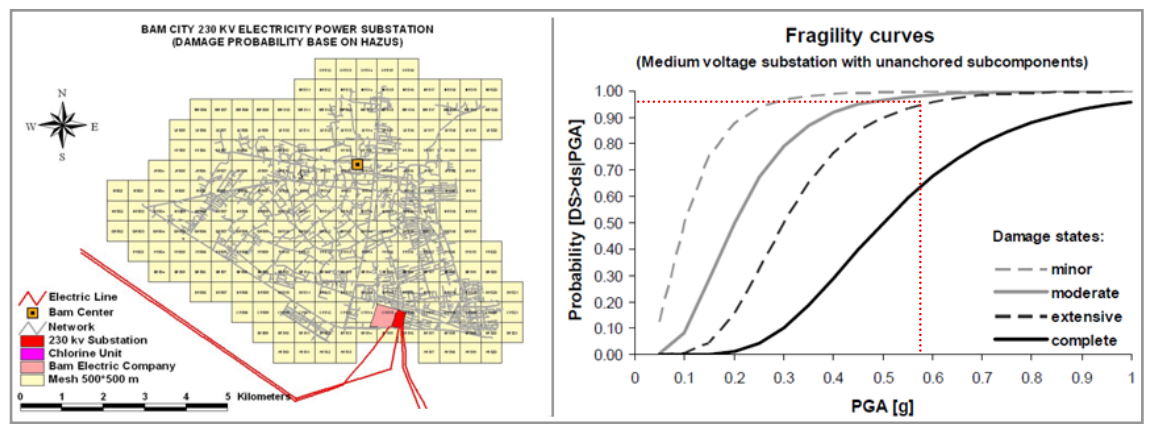

Figure 10: Bam $230 \mathrm{kv}$ electric substation and HAZUS fragility curve.

In fact, the bam substation is damaged in this hazard level (475 years). Although, this level of ground shaking has been used for designing ordinary buildings in high seismic areas and in case of higher importance facilities such as hospitals and lifeline infrastructures must be designed for hazard events with higher return period than 475 years. Hence Bam electric substation was extensive damaged inevitably due to 2003 earthquake, fig. 1 . 


\section{Conclusion}

Given the importance of lifeline systems to the society; reliable seismic assessment of those systems becomes crucial for better preparedness and disaster management. Modeling the seismic response of is one approach towards more accurate anticipation of the effects of earthquakes in the urban areas. This paper summarizes the framework of lifeline seismic analysis especially PSHA methodology and revises the probabilistic seismic hazard maps of Bam city. After considering the active fault and considering appropriate attenuation relationships, PGA and PGV were calculated in 475-year return period for $500 \mathrm{~m}$ grid mesh. The repair ratio of buried pipelines is computed for each cell based on ALA-2001, as an earthquake performance of water network. According to the location of the Bam electric substation, the probability of physical damage is about 0.96 , therefore is damaged in 475 years hazard level. The results consistent with happened damages after the earthquake. Districts experiencing a high level of distribution network damage also experienced a high level of building damage, therefore it seems that in case of network input data lack and without any lifeline damage assessment analysis, using the building damage database prepare suitable rough network damage estimation for disaster management and post earthquake activities such as emergency water supply.

\section{References}

[1] Anderson, J.G., Brune, J.N., Anooshehpoor, R., Ni, S.-D., New ground motion data and concepts in seismic hazard analysis. Current Science, 79, pp. 1278-1289, 2000.

[2] Baker, J. W., and Jayaram, N., Correlation of spectral acceleration values from NGA ground motion models. Earthquake Spectra, 24, pp. 299-317, 2008.

[3] EEFIT, The Bam, Iran Earthquake of December 26, 2003, The Institution of Structural Engineers, Earthquake Engineering Field Investigation Team.

[4] EERI, Preliminary observations on the Bam, Iran, Earthquake of December 26, 2003 Learning From Earthquakes, Earthquake Engineering Research Institute, Special Report, 2004.

[5] FEMA. HAZUS-MH technical manual: earthquake model. Multi Hazard Loss Estimation Methodology. United States Department of Homeland Security, Federal Emergency Management Agency, Washington, DC, 2003.

[6] Ghodrati Amiri, G., Mahdavian, A. and Manouchehri Dana, F. Attenuation Relation for Iran. Journal of Earthquake Engineering, 11, pp. 469-492, 2007.

[7] Hessami K, Jamali F. and Tabassi H., Major Active Faults of Iran, Seismotectonic Department, International Institute of Earthquake Engineering and Seismology (IIEES), Iran, 2003.

[8] O'Rourke T.D., Critical infrastructure, interdependencies, and resilience. Bridge, National Academy of Engineering, 37(1):22-29, 2007. 